\title{
Evaluation of SSR and SNP Markers for Molecular Breeding in Rice
}

\author{
Zennia Jean Gonzaga ${ }^{1}$, Kashif Aslam ${ }^{1,2}$, Endang M. Septiningsih ${ }^{1}$, Bertrand C.Y. Collard ${ }^{1}$ * \\ ${ }^{1}$ Plant Breeding, Genetics and Biotechnology (PBGB), International Rice Research Institute (IRRI), DAPO 7777, Metro Manila, \\ Philippines \\ ${ }^{2}$ Institute of Molecular Biology and Biotechnology (IMBB), Bahauddin Zakariya University, Multan Pakistan
}

\begin{abstract}
Simple sequence repeats (SSRs) have been the marker of choice for rice molecular breeding due to the high level of polymorphism, technical simplicity and low cost. Recent advances in rice genomics have led to the discovery of abundant single nucleotide polymorphism (SNPs) which have enormous potential for rice molecular breeding. To assess both marker systems for molecular breeding in rice, SSR and SNP markers were evaluated on a set of 23 genotypes representing indica germplasm for their usefulness in molecular research and breeding program. Seven hundred SSR and sequence tagged sites (STS) markers and 384 SNPs were screened for polymorphism. Highly polymorphic markers based on polymorphic information content (PIC) values were identified, which will be useful for molecular breeding. Data was used to identify an "indica genotyping set" based on high level of polymorphism, chromosome position and marker quality which will provide kits of markers for marker assisted selection (MAS). Genetic diversity analysis using SSR data was more consistent with pedigrees compared to analysis with SNP data indicating that more than 384 SNPs are required when elite indica breeding material is used. The results also indicated that there were polymorphic "blind spots" for the fixed SNP set suggesting that SSRs could still be used to complement fixed-SNP genotyping platforms for some molecular breeding applications.
\end{abstract}

Keywords Molecular breeding, Genotyping set, PIC, SSR, STS, SNP

\section{INTRODUCTION}

Rice (Oryza sativa L.) is the most important crop in Asia. Many constraints to rice production may be overcome by developing new rice varieties (e.g. breeding for biotic or abiotic stress tolerance). Molecular breeding using DNA markers can greatly increase the precision and efficiency of rice breeding and therefore may provide useful tools for breeders (Jena and Mackill 2008). Marker assisted selection (MAS) is advantageous over conventional phenotypic selection for the following reasons: it may be simpler than phenotypic screening which can save time, resources and effort; selection can be carried out at the seedling stage; and single plants can be accurately selected (Collard and Mackill 2008; Ribaut and Hoisington 1998). Markerassisted backcrossing (MABC), a specific MAS scheme in plant molecular breeding, has been used to upgrade new varieties for many traits, especially for abiotic stress tolerance in rice (Gregorio et al. 2013; Mackill et al. 2012).

Of the many types of DNA markers, microsatellites or simple sequence repeats (SSRs) are widely used due to their high polymorphism and technically simple method of detection (Wu and Tanksley 1993; Panaud et al. 1996; Akagi et al. 1997; Chen et al. 1997; McCouch et al. 2002). SSRs, like other PCR-based markers, can be run with common lab equipment and are cost effective (for low throughput). In rice, they have been extremely well characterized largely due to the completion of the rice genome reference sequence from Nipponbare (MSU7) and the availability of numerous genomics resources (Schatz et al. 2014). Hence, SSRs have been the marker of choice for rice molecular breeding. The disadvantages of SSRs include

Received May 31, 2015; Revised Jun 17, 2015; Accepted Jun 19, 2015; Published June 30, 2015

*Corresponding author Bertrand C.Y.Collard, b.collard@irri.org, bcycollard@hotmail.com, Tel: +63-2-580-5600-2478 
the requirement of polyacrylamide gel electrophoresis, limited abundance when saturating genomic regions, constraints in multiplexing, limitations in universal data storage, and automation. Moreover, SSRs may differ in robustness, quality of the amplification products, and amplification of single or multiple loci which suggests that not all SSRs are equal. They may have low information content in the gene of interest (Macaulay et al. 2001).

In recent years, single nucleotide polymorphisms (SNPs) have become widely used DNA markers because they are more efficient, stable and cost effective (for high throughput) (Rafalski 2002; Duran et al. 2009; Edwards and Batley 2010). Moreover, automated, high-throughput processing of large numbers of samples is possible. SNPs are the most abundant variations in the genome that are ideal for high-resolution genotyping, hence, they are appropriate for association mapping, genetic diversity analysis, linkage mapping and MAS (McCouch et al. 2010; Tung et al. 2010). With the development of genotyping by sequencing (GBS), SNPs are expected to remain as the marker of choice in the $21^{\text {st }}$ century (Thomson 2014).

Recently in rice, Narshimulu et al. (2011) provided "ready-to-use" SSR markers for background selection for marker-assisted breeding in rice. A total of 840 hypervariable microsatellite markers (hvRMs) evenly distributed in the genome were selected from 18,828 Class I SSRs (hypervariable markers that are more polymorphic than Class II due to longer repeats of $\geq 20$ nucleotides in length as reported by Cho et al. (2000) and Temnykh et al. (2000)). A selection of 36 hvRMs were selected from the 840 markers and screened on 24 diverse rice cultivars to estimate the polymorphic information content (PIC). PIC is a measure of polymorphism for a marker locus (Shete et al. 2000). It considers both the number of alleles that are expressed and the relative frequencies of those alleles in order to provide an estimate of the discriminatory power of a locus. The values ranged from 0 (monomorphic) to 1 (highly discriminative) (Anderson et al. 1993). A "MAS kit" of molecular markers was developed using the hvRMs that are tightly-linked to important agronomic traits to effectively speed up the process of crop improvement. Singh et al. (2009) evaluated the relationship between SSR length and level of polymorphism in eight diverse rice genotypes using 201 random SSR loci to find most variable SSR loci for agarose gel electrophoresis.

Previous studies in assessing genetic relatedness in maize association mapping panels confirmed that SSR markers with moderate density were more informative than SNPs (Yang et al. 2011). Although, there was a high coefficient of correlation between the genetic similarity of SSR and SNP genotyping data, average PIC values of SSRs in barley were higher compared to SNP markers (Varshney et al. 2008; 2010). High throughput SNP genotyping platforms are becoming more accessible and hence replacing SSR markers in molecular breeding research. Thomson et al. (2012) designed seven GoldenGate VeraCode oligo pool assay (OPA) sets (384 SNPs) for rice using the Illumina BeadXpress platform. The results indicated that 384-plex SNP genotyping using the BeadXpress platform was effective for diversity analysis, QTL mapping, MABC and developing specialized genetic (Thomson et al. 2012).

The aim of this study was to evaluate a large number of SSRs and SNPs using an important set of indica varieties or important donor parents which are highly relevant to current breeding activities at the International Rice Research Institute (IRRI). Our main objective was to identify highly polymorphic SSRs and SNPs that could be used as "genotyping sets" in rice for various molecular breeding applications, as have been reported in other crops such as barley (Macaulay et al. 2001) or cotton (Lacape et al. 2007). In many previous studies, a diverse set of genotypes is often used including indica and japonica subspecies, which may give an exaggerated indication of the potential of markers for breeding applications. Hence our focus was on indica germplasm, which is the predominant sub-species in South and Southeast Asia. Particular emphasis was given to breeding for submergence tolerance by focusing on the SUB1 locus on chromosome 9 (Xu and Mackill 1996). SUB1 is a major QTL controlling submergence tolerance and has been used to develop 'upgraded' rice mega-varieties (Iftekharuddaula et al. 2011; Mackill et al. 2012; Neeraja et al. 2007; Septiningsih et al. 2009, 2013, 2015). 


\section{MATERIALS AND METHODS}

\section{Plant material}

A total of 23 genotypes including important irrigated and rainfed indica varieties, donor parents and elite breeding lines were assembled, with an emphasis on breeding for flood tolerance at IRRI. Many SUB1 donors (i.e. released $S U B 1$ varieties) and selected recipient parents were specifically included in order to identify polymorphic markers for current research and molecular breeding activities. Parents used in mapping populations for submergence and stagnant flooding tolerance were also included (Table 1). Additional

Table 1. Rice genotypes used for marker evaluation.

\begin{tabular}{|c|c|c|c|c|c|}
\hline Name & Alias & Parentage & Designation & GID & Information \\
\hline Swarna-Sub1 & & Swarna*4/IR49830-7-1-2-3 & IR05F102 & 1847271 & $\begin{array}{l}\text { New variety with submergence } \\
\text { tolerance in India }\end{array}$ \\
\hline $\begin{array}{l}\text { Samba } \\
\text { Mahsuri-Sub1 }\end{array}$ & & $\begin{array}{l}\text { SAMBHA MAHSURI*3/IR } \\
49830-7-1-2-3\end{array}$ & IR07F101 & 2159598 & $\begin{array}{l}\text { New variety with submergence } \\
\text { tolerance in India }\end{array}$ \\
\hline BR11-Sub1 & $\begin{array}{l}\text { BRRI dhan } 52 \\
\text { (Bangladesh) }\end{array}$ & BR $11 * 3 /$ IR $40931-33-1-3-2$ & IR07F290 & 2295328 & $\begin{array}{l}\text { New variety with submergence } \\
\text { tolerance in Bangladesh }\end{array}$ \\
\hline CR1009-Sub1 & & CR 1009*3/IR 49830-7-1-2-3 & IR07F291 & 2403712 & $\begin{array}{l}\text { New variety with submergence } \\
\text { tolerance in India }\end{array}$ \\
\hline IR09F434 & & IRRI $123 * 2 / I R R I ~ 149$ & IR09F434 & 2847875 & $\begin{array}{l}\text { Sub1 near isogenic line (NIL) in } \\
\text { popular Philippine variety PSBRc } 82\end{array}$ \\
\hline Ciherang-Sub1 & Inpari 30 (Indonesia) & CIHERANG*2/IRRI 149 & IR09F436 & 2847870 & $\begin{array}{l}\text { New variety with submergence } \\
\text { tolerance in Indonesia }\end{array}$ \\
\hline PSBRc18-Sub1 & & $\begin{array}{l}\text { PSB RC } 18(\text { IR } \\
51672-62-2-1-1-2-3) * 2 / \text { IRRI } 149\end{array}$ & IR09F437 & 2853229 & $\begin{array}{l}\text { Sub1 near isogenic line (NIL) in } \\
\text { popular Philippine variety awaiting } \\
\text { to be released }\end{array}$ \\
\hline IR64-Sub1 & $\begin{array}{l}\text { IRRI } 149 \text { or } \\
\text { NSICRc194 or } \\
\text { "Submarino" } \\
\text { (Philippines) }\end{array}$ & IR 40931-33-1-3-2/3*IR 64 & IR07F102 & 2159583 & $\begin{array}{l}\text { New variety with submergence } \\
\text { tolerance in the Philippines }\end{array}$ \\
\hline Sabitri & & IR 1561-228-1/IR 1737//CR 94-13 & IRTP 8487 & 2268099 & Popular variety in Nepal \\
\hline IR6 & & $\begin{array}{l}\text { SIAM } 29(\text { ACC 42)/DEE GEO } \\
\text { WOO GEN }\end{array}$ & IRTP 25398 & 2440173 & Popular variety in Pakistan \\
\hline Super basmati & & BAS 320/IR 661 & IRTP 20918 & 2274228 & Popular basmati variety in Pakistan \\
\hline IR09F185 & & $\begin{array}{l}\text { BR 11/IR } \\
49830-7-1-2-3 / / I R 04 N 106\end{array}$ & $\begin{array}{l}\text { IR } 85288-S U B \\
38-1-1\end{array}$ & 2707581 & $\begin{array}{l}\text { Stagnant flooding donor. Parent of } \\
\text { RIL population }\end{array}$ \\
\hline $\begin{array}{l}\text { IR67440-NDR-5-1- } \\
1-1-1\end{array}$ & & CNM 539/IR 53479-B-45-3-2-3 & & 84299 & $\begin{array}{l}\text { Stagnant flooding donor. Parent of } \\
\text { RIL population }\end{array}$ \\
\hline FR13A & & DHALPUTTIA & & 32293 & $\begin{array}{l}\text { Donor of Sub1 and highly flood } \\
\text { tolerant landrace }\end{array}$ \\
\hline IR40931 & & $\begin{array}{l}\text { BKNFR 76106-16-0-1/IR } \\
19661-131-1-2\end{array}$ & IR40931-33-1-3-2 & 71615 & Sub1 donor parent. \\
\hline IR49830 & “Popoul” (Myanmar) & $\begin{array}{l}\text { IR } 4568-86-1-3-2 / \text { IR } \\
26702-111-1 / / \text { IR } \\
20992-7-2-2-2-2-3 / \text { IR } \\
21567-9-2-2-2-1\end{array}$ & IR49830-7-1-2-3 & 88474 & $\begin{array}{l}\text { Sub1 and stagnant flooding donor } \\
\text { parent/variety }\end{array}$ \\
\hline IR42 & & $\begin{array}{l}\text { IR } 1561-228-1-2 / \text { IR } 1737 / / C R \\
94-13\end{array}$ & IR 2071-586-5-6 & 13988 & $\begin{array}{l}\text { IRRI variety released for irrigated } \\
\text { and rainfed areas. Susceptible check } \\
\text { for submergence tolerance screening. }\end{array}$ \\
\hline IRBB66 & & $\begin{array}{l}\text { IRBB 7/IR BB } 60 \text { (IR } \\
72920-1-44-4)\end{array}$ & & 1847199 & $\begin{array}{l}\text { Widely-used bacterial blight gene } \\
\text { donor (Xa4, xa5, xa7, xa13 and } \\
\text { Xa21) in IR24 background }\end{array}$ \\
\hline OR $142-99$ & $\begin{array}{l}\text { Santepheap } 3 \\
\text { (Cambodia) }\end{array}$ & PANKAIJ/SIGADIS & IRTP 13636 & 415260 & $\begin{array}{l}\text { Indian breeding line released as a } \\
\text { variety in Cambodia }\end{array}$ \\
\hline IRRI 119 & $\begin{array}{l}\text { PSBRc68 or } \\
\text { 'Sacobia' } \\
\text { (Philippines); Shwe } \\
\text { Pyi Tan (Myanmar) }\end{array}$ & $\begin{array}{l}\text { IR } 43581-57-3-3-6 / \text { IR } \\
\text { 26940-20-3-3-3-1//KHAO DAWK } \\
\text { MALI } 105\end{array}$ & PSB RC 68 & 2266161 & $\begin{array}{l}\text { IRRI variety released with Sub1 for } \\
\text { rainfed areas in the Philippines with } \\
\text { stagnant flooding tolerance }\end{array}$ \\
\hline IRRI 154 & $\begin{array}{l}\text { NSICRc222 or } \\
\text { 'Tubigan 18' } \\
\text { (Philippines) }\end{array}$ & $\begin{array}{l}\text { IR 73012-137-2-2-2/PSB RC } 10 \\
\text { (IR 50404-57-2-2-3) }\end{array}$ & IR04A412 & 1253989 & $\begin{array}{l}\text { IRRI variety released for irrigated } \\
\text { and favourable rainfed areas in the } \\
\text { Philippines }\end{array}$ \\
\hline FL478 & IR 66946-3R-178-1-1 & IR 29/POKKALI B & $\begin{array}{l}\text { IR } \\
66946-3 R-178-1-1\end{array}$ & 1192884 & $\begin{array}{l}\text { Widely used salinity tolerant donor } \\
\text { line }\end{array}$ \\
\hline IRRI 148 & $\begin{array}{l}\text { NSICRc192 or } \\
\text { 'Sahod Ulan } 1 \text { ' } \\
\text { (Philippines) }\end{array}$ & IR 55419-4*2/WAY RAREM & IR74371-54-1-1 & 1161411 & $\begin{array}{l}\text { Drought tolerant IRRI variety } \\
\text { released in the Philippines }\end{array}$ \\
\hline
\end{tabular}


information about these donor parents was described in Collard et al. (2013) and Mackill et al. (1993).

\section{DNA Extraction}

DNA was isolated using modified mini prep CTAB method (Zheng et al. 1995). Young leaves of 2 week old plants were sampled and ground using Genogrinder (SPEX SamplePrep, Metuchen, NJ, USA) equipment and liquid nitrogen. Addition of $2 \%$ CTAB buffer $(100 \mathrm{mM}$ Tris- $\mathrm{HCl}$ pH8, $50 \mathrm{mM}$ EDTA $\mathrm{pH}=8,500 \mathrm{mM} \mathrm{NaCl}, 1.25 \%$ SDS, $0.38 \%$ sodium biosulfate) and incubation in water bath at $65^{\circ} \mathrm{C}$ for 1 hour followed. Subsequently, the mixture was shaken sporadically, followed by the treatment with chloroform:isoamyl alcohol mixture (24:1) to remove protein. Afterwards, supernatant was precipitated in ice cold isopropanol for 1 hour and pellets were washed with 70\% ethanol. RNA was removed using RNase and pellets were dissolved in TE buffer. Finally, the quality of DNA was verified using $1 \%$ agarose gel stained with $\operatorname{Sybr}{ }^{\circledR}$ safe (Invitrogen, Catalog no. S33102) and DNA was quantified using Nano Drop Spectrophotometer (Thermo Scientific, Wilmington, DE, USA). The DNA samples were normalized to $20 \mathrm{ng} / \mathrm{uL}$ and $50 \mathrm{ng} / \mathrm{uL}$ for SSR and SNP genotyping, respectively.

\section{SSR genotyping}

Seven hundred SSR and STS markers obtained from Genetics and Genomics Laboratory at IRRI were selected across all 12 rice chromosomes. Most SSRs were selected based on previous research identifying these SSRs to have high PIC values, whereas others were based on the availability in the laboratory. Other SSR or STS markers were selected based on the physical position near SUBI (e.g. ART5, RM3609). PCR amplification was performed in $7 \mathrm{uL}$ volume containing of $20 \mathrm{ng} / \mathrm{uL}$ DNA (2uL) template, 10x PCR buffer $(0.7 \mathrm{uL}), 1 \mathrm{mM}$ dNTPs $(0.7 \mathrm{uL})$, $1.5 \mathrm{mM} \mathrm{MgCl}_{2}(0.6 \mathrm{uL}), 5 \mathrm{uM}$ of each forward and reverse primer ( $0.4 \mathrm{uL}$ each) and 1 unit of homemade Taq DNA polymerase $(0.9 \mathrm{uL})$ on thermal cycler (G-Storm, United Kingdom). The temperature profile used for amplification includes: 3 min of initial denaturation at $94^{\circ} \mathrm{C}$ followed by 35 cycles of denaturation at $94^{\circ} \mathrm{C}$ for $30 \mathrm{sec}$, annealing at $54-55^{\circ} \mathrm{C}$ for $30 \mathrm{sec}$ and extension at $72^{\circ} \mathrm{C}$ for $30 \mathrm{sec}$, and the final extension at $72^{\circ} \mathrm{C}$ for $2 \mathrm{~min}$. A no DNA template control was used to determine if there was contamination within the process. The samples were then mixed with $2 \mathrm{uL}$ of bromophenol blue gel loading dye and $4 \mathrm{uL}$ PCR products were separated using $8 \%$ polyacrylamide gel along with a $1 \mathrm{~kb}$ ladder (Invitrogen, Cat No.10787026) for 2 hours. Visualization was done using Alpha Imager 1220 (Alpha Innotech, CA, USA) under ultraviolet (UV) light after staining with Sybr ${ }^{\circledR}$ safe (Invitrogen, Catalog no. S33102). The amplicons were scored manually in base pairs using the $1 \mathrm{~kb}$ ladder. The protocol was optimized for SSRs that failed once and repeated for 2 or 3 times. After the third attempt, SSRs that still failed to amplify were not used again. Genetic and physical map positions were determined using the Gramene website (www.gramene.org) and from the Nipponbare rice genome reference (IRGSP, 2005). Marker 'quality' was assessed and defined as the clarity and reproducibility of the marker.

\section{SNP genotyping}

A 384-plex SNP genotyping assay on the BeadXpress (Illumina ${ }^{\circledR}$ ) platform was used. Normalized DNA samples were genotyped using "GoldenGate Genotyping Assay" (VeraCode Manual Protoco; Illumina Part 311275211), following the manufacturer's instructions. An Allegra 25R (Beckman Coulter, Brea, CA, USA) and GSI thermal cycler (G-storm, Surrey, UK) were used for the plate centrifuge steps and for PCR amplification respectively. RiceOPA2.1 was used for genotyping the 23 rice varieties in which the Illumina OPA ID was GS0011861-OPA ${ }^{\mathrm{a}}$. This OPA is appropriate for indica genotypes and informative for indica/indica populations (Thomson et al. 2012).

\section{Data analysis}

The size of SSR marker alleles were estimated and scored based on the $1 \mathrm{~kb}$ ladder (10787-018, Life Technologies). SSR data in base pairs was directly inputted in the PowerMarker software (v3.25). The SNP data was analyzed using the Genotyping module (v1.6.3) of the Illumina GenomeStudio (v2010.1) software. For a more accurate allele calling, ALCHEMY program was used because it 
was created for highly inbred samples (Thomson et al. 2012). The ALCHEMY-Illumina plug-in v1.0 (http:// alchemy.sourceforge.net/) was installed into GenomeStudio. The Genome Studio "Reports Wizard" was used to output the data into ALCHEMY format resulting to two data, one with the project name containing sample intensity data and the second as a map file with the SNP map information. The outputs were used as input files for ALCHEMY and all succeeding output re-shaping and manipulations was done in the GALAXY-ALCHEMY bioinformatics workflow environment (http://main.g2.bx.psu.edu/). Allele calls were used for the genetic diversity analysis using Nei1983 genetic distance measure with a neighbor-joining tree with PowerMarker v3.25 software (Liu and Muse 2005; http:// statgen.ncsu.edu/powermarker/). Bootstrap NJ phylogenetic trees were produced using TreeView (http://taxonomy. zoology.gla.ac.uk/rod/treeview.html). PIC values were calculated using PowerMarker based on the formula reported by Botstein et al. (1980). The PIC values of 23 genotypes (including FR13A and Super Basmati) were compared with 21 genotypes (excluding FR13A and Super Basmati), however PIC values based on only the 21 indica genotypes were shown in Tables 2 and 3. Polymorphism survey data can be obtained from the corresponding author upon request.

\section{RESULTS}

\section{SSR genotyping}

Four hundred ninety out of 722 SSRs tested (68\%) were polymorphic across the 23 genotypes used. In terms of polymorphism, chromosome 5 had the highest percentage of $80 \%$ and chromosome 11 had the lowest percentage of $52.8 \%$. However, chromosome 11 had the highest maximum PIC value of 0.83 and chromosome 12 had the lowest value of 0.563 . The average PIC values ranged from 0.331 to 0.461 (Table 2). SSR genotyping set was created based on the reliability of the marker measured visually through the band quality, polymorphism using the generated PIC values and marker position with at least $2 \mathrm{Mb}$ distance between each markers (Table 3). Chromosome 9, the location of SUBI was examined for tightly linked SSR markers between 5.92-8 Mb. SUB1 marker alleles were recorded for each marker across the 23 genotypes (Table 4).

\section{SNP genotyping}

Three hundred sixty five out of 384 SNPs screened (95\%) were polymorphic across 23 genotypes which was $27 \%$ higher than SSRs. Chromosomes 5, 8 and 9 had 100\% polymorphism while chromosome 3 had the lowest polymorphism of $86.4 \%$ which was still higher by $33.6 \%$ of the lowest value in SSR. However, the maximum PIC value of SSRs (0.563-0.830) was higher compared with SNPs

Table 2. Polymorphic SSR markers and PIC values per chromosome.

\begin{tabular}{|c|c|c|c|c|c|c|}
\hline Chr & No. SSRs & $\%$ monomorphic & $\%$ polymorphic & PIC Min & PIC Max & PIC Ave \\
\hline 1 & 107 & 35.2 & 64.8 & 0.087 & 0.720 & 0.338 \\
\hline 2 & 64 & 25.4 & 74.6 & 0.087 & 0.691 & 0.333 \\
\hline 3 & 60 & 27.1 & 72.9 & 0.087 & 0.648 & 0.331 \\
\hline 4 & 61 & 35.0 & 65.0 & 0.087 & 0.748 & 0.332 \\
\hline 5 & 56 & 20.0 & 80.0 & 0.087 & 0.735 & 0.340 \\
\hline 6 & 53 & 24.0 & 76.0 & 0.087 & 0.791 & 0.386 \\
\hline 7 & 38 & 26.3 & 73.7 & 0.087 & 0.813 & 0.402 \\
\hline 8 & 52 & 25.0 & 75.0 & 0.087 & 0.711 & 0.396 \\
\hline 9 & 102 & 24.7 & 75.3 & 0.087 & 0.772 & 0.400 \\
\hline 10 & 33 & 37.5 & 62.5 & 0.087 & 0.703 & 0.414 \\
\hline 11 & 42 & 47.2 & 52.8 & 0.087 & 0.830 & 0.461 \\
\hline 12 & 54 & 37.3 & 62.7 & 0.087 & 0.563 & 0.352 \\
\hline
\end{tabular}


Table 3. Indica SSR genotyping set based on reliability, polymorphism and marker position.

\begin{tabular}{|c|c|c|c|c|c|c|c|}
\hline SSR Name & Chr. & Physical (Mb) & PIC Values & SSR Name & Chr. & Physical (Mb) & PIC Values \\
\hline RM495 & 1 & 0.2 & 0.4393 & RM400 & 6 & 28.4 & 0.6172 \\
\hline RM1 & 1 & 4.6 & 0.2608 & RM51 & 7 & 0.2 & 0.4368 \\
\hline RM243 & 1 & 7.9 & 0.3975 & RM481 & 7 & 2.8 & 0.8129 \\
\hline RM582 & 1 & 9.1 & 0.4470 & RM21077 & 7 & 4 & 0.0866 \\
\hline RM449 & 1 & 15.1 & 0.1575 & RM214 & 7 & 12.7 & 0.3967 \\
\hline RM24 & 1 & 18.9 & 0.5334 & RM500 & 7 & 15.9 & 0.3698 \\
\hline RM237 & 1 & 26.8 & 0.4345 & RM2 & 7 & 16 & 0.3457 \\
\hline RM84 & 1 & 29.7 & 0.3554 & RM320 & 7 & 18.6 & 0.6175 \\
\hline RM486 & 1 & 34.9 & 0.3249 & RM336 & 7 & 21.8 & 0.7137 \\
\hline RM14 & 1 & 41.3 & 0.5885 & RM18 & 7 & 25.6 & 0.4252 \\
\hline RM154 & 2 & 1 & 0.6913 & RM248 & 7 & 29.3 & 0.6398 \\
\hline RM279 & 2 & 2.8 & 0.5468 & RM6925 & 8 & 0.6 & 0.6608 \\
\hline RM71 & 2 & 8.7 & 0.5623 & RM38 & 8 & 2.1 & 0.4303 \\
\hline RM324 & 2 & 11.3 & 0.4394 & RM25 & 8 & 4.3 & 0.4160 \\
\hline RM300 & 2 & 13.1 & 0.4925 & RM72 & 8 & 6.7 & 0.6494 \\
\hline RM341 & 2 & 19.3 & 0.5192 & RM3395 & 8 & 10.2 & 0.7069 \\
\hline RM263 & 2 & 25.8 & 0.5109 & RM22837 & 8 & 12.3 & 0.7109 \\
\hline RM1342 & 2 & 28.1 & 0.5396 & RM404 & 8 & 15.4 & 0.6024 \\
\hline RM6 & 2 & 29.5 & 0.3457 & RM223 & 8 & 20.6 & 0.4368 \\
\hline RM208 & 2 & 35.1 & 0.5743 & RM52 & 8 & 24.7 & 0.5109 \\
\hline RM231 & 3 & 2.4 & 0.5736 & RM5545 & 8 & 28.2 & 0.5425 \\
\hline RM218 & 3 & 8.4 & 0.6479 & RM23679 & 9 & 0.8 & 0.4783 \\
\hline RM3297 & 3 & 13.2 & 0.3131 & RM23793 & 9 & 4.3 & 0.5603 \\
\hline RM15187 & 3 & 16.6 & 0.4551 & RM5515 & 9 & 7.1 & 0.4925 \\
\hline RM411 & 3 & 21.4 & 0.3604 & RM23958 & 9 & 7.9 & 0.4394 \\
\hline RM426 & 3 & 27.5 & 0.3850 & RM3855 & 9 & 9.3 & 0.4160 \\
\hline RM186 & 3 & 28.8 & 0.5736 & RM24087 & 9 & 10.8 & 0.5109 \\
\hline RM571 & 3 & 33.1 & 0.2970 & RM105 & 9 & 12.5 & 0.5267 \\
\hline RM570 & 3 & 35.5 & 0.4647 & RM24260 & 9 & 14.1 & 0.3554 \\
\hline RM85 & 3 & 36.3 & 0.5038 & RM410 & 9 & 17.6 & 0.4818 \\
\hline RM335 & 4 & 0.6 & 0.6550 & RM242 & 9 & 18.8 & 0.4783 \\
\hline RM261 & 4 & 6.5 & 0.4065 & RM108 & 9 & 19.3 & 0.3457 \\
\hline RM307 & 4 & 13.1 & 0.5623 & RM205 & 9 & 22.7 & 0.3850 \\
\hline RM16945 & 4 & 20.5 & 0.2373 & RM24888 & 10 & 0.5 & 0.3554 \\
\hline RM3839 & 4 & 23.9 & 0.2970 & RM222 & 10 & 2.6 & 0.5015 \\
\hline RM252 & 4 & 25.1 & 0.5509 & RM216 & 10 & 5.3 & 0.4842 \\
\hline RM3820 & 4 & 27.6 & 0.4898 & RM25436 & 10 & 14.9 & 0.5578 \\
\hline RM303 & 4 & 28.5 & 0.2922 & RM25459 & 10 & 15.2 & 0.5780 \\
\hline RM5473 & 4 & 31.4 & 0.7481 & RM258 & 10 & 18 & 0.5880 \\
\hline RM280 & 4 & 34.9 & 0.4638 & RM228 & 10 & 22.2 & 0.6801 \\
\hline RM13 & 5 & 2 & 0.5396 & RM286 & 11 & 0.3 & 0.5877 \\
\hline RM592 & 5 & 2.7 & 0.7349 & RM167 & 11 & 4 & 0.2149 \\
\hline RM17954 & 5 & 3.6 & 0.5425 & RM202 & 11 & 9 & 0.3967 \\
\hline RM18188 & 5 & 9.1 & 0.6657 & RM26664 & 11 & 15.3 & 0.8295 \\
\hline RM1115 & 5 & 14.7 & 0.5109 & RM209 & 11 & 17.8 & 0.6982 \\
\hline RM5454 & 5 & 17.8 & 0.6221 & RM26834 & 11 & 18.6 & 0.5736 \\
\hline RM163 & 5 & 19.1 & 0.5509 & RM21 & 11 & 19.1 & 0.6769 \\
\hline RM3575 & 5 & 21.3 & 0.4160 & RM206 & 11 & 22 & 0.6641 \\
\hline RM274 & 5 & 26.8 & 0.0866 & RM224 & 11 & 27.2 & 0.3554 \\
\hline RM31 & 5 & 28.6 & 0.4551 & RM27389 & 11 & 28.4 & 0.6690 \\
\hline RM435 & 6 & 0.5 & 0.3967 & RM3472 & 12 & 3.5 & 0.5578 \\
\hline RM586 & 6 & 1.4 & 0.6272 & RM27809 & 12 & 7.4 & 0.3744 \\
\hline RM204 & 6 & 3.1 & 0.7913 & RM27933 & 12 & 10.4 & 0.3975 \\
\hline RM3408 & 6 & 4.5 & 0.3249 & RM28102 & 12 & 16 & 0.4303 \\
\hline RM276 & 6 & 6.2 & 0.3604 & RM1261 & 12 & 17.5 & 0.5629 \\
\hline RM549 & 6 & 6.9 & 0.3604 & RM415 & 12 & 19.5 & 0.3026 \\
\hline RM136 & 6 & 8.7 & 0.2149 & RM17 & 12 & 26.9 & 0.4813 \\
\hline RM3827 & 6 & 22.2 & 0.5179 & RM28825 & 12 & 27.5 & 0.2373 \\
\hline RM30 & 6 & 27.2 & 0.5921 & & & & \\
\hline
\end{tabular}


Table 4. SSR markers tightly linked to SUB1.

\begin{tabular}{|c|c|c|c|c|c|c|c|c|c|c|}
\hline Genotype & & & & & Chromo & ome $9^{\mathrm{a}, \mathrm{b}}$ & & & & \\
\hline Marker & RM8206 & ART5 & RM464 & RM8300 & RM6920 & RM5515 & RM5526 & RM219 & SC30 & RM23958 \\
\hline Physical position $(\mathrm{Mb})$ & 5.92 & 6.30 & 6.58 & 6.60 & 7.01 & 7.15 & 7.31 & 7.89 & 8.00 & 8.00 \\
\hline PIC Value & 0.157 & 0.484 & 0.425 & 0.325 & 0.537 & 0.493 & 0.374 & 0.527 & 0.568 & 0.439 \\
\hline Swarna-Sub1 & 152 & 200 & 262 & 200 & 300 & $\mathrm{n} / \mathrm{a}$ & 172 & 202 & 178 & 98 \\
\hline Samba Mahsuri-Sub1 & 152 & 200 & 262 & 200 & 300 & 124 & 170 & 206 & 178 & 80 \\
\hline CR1009-Sub1 & 152 & 200 & 262 & 200 & 300 & 124 & 172 & 208 & 184 & 98 \\
\hline BR11-Sub1 & 152 & 200 & 262 & 200 & 250 & 126 & 172 & 204 & 178 & 98 \\
\hline PSBRc82-Sub1 & 152 & 200 & 262 & 200 & 300 & 126 & 172 & 204 & 170 & 80 \\
\hline Ciherang-Sub1 & 152 & 200 & 262 & 200 & 300 & 126 & 170 & 206 & 170 & 80 \\
\hline PSBRc18-Sub1 & 154 & 200 & 262 & 200 & 250 & 126 & 170 & 206 & 190 & 98 \\
\hline IR64-Sub1 & 152 & 200 & 262 & 200 & 300 & 126 & 170 & 206 & 178 & 80 \\
\hline Sabitri & 152 & 210 & 262 & 198 & 300 & 124 & 170 & 206 & 170 & 80 \\
\hline IR6 & 152 & 210 & 300 & 200 & 250 & 124 & 172 & 208 & 178 & 80 \\
\hline Super basmati & 158 & 210 & 258 & 200 & 300 & 128 & 172 & 206 & 190 & 82 \\
\hline IR09F185 & 152 & 200 & 262 & 200 & 300 & 126 & 172 & 206 & 178 & 80 \\
\hline IR67440-NDR-5-1-1-1-1 & 152 & 210 & 262 & 198 & 250 & 124 & 170 & 206 & 170 & 80 \\
\hline FR13A $A^{b}$ & 152 & 200 & 262 & 200 & 300 & 126 & 172 & 202 & 178 & 82 \\
\hline IR40931 & 152 & 200 & 262 & 200 & 250 & 126 & 172 & 202 & 178 & 82 \\
\hline IR49830 & 152 & 200 & 262 & 200 & 300 & 124 & 170 & 206 & 170 & 80 \\
\hline IR42 & 152 & 210 & 262 & 198 & 290 & 124 & 170 & 206 & 170 & 80 \\
\hline IRRBB66 & 152 & 210 & 300 & 200 & 290 & 124 & 170 & 206 & 178 & 80 \\
\hline OR $142-99$ & 152 & 210 & 270 & 198 & 250 & 124 & 172 & 208 & 178 & 98 \\
\hline IRRI 119 & 152 & $\mathrm{n} / \mathrm{a}$ & $\mathrm{n} / \mathrm{a}$ & 200 & 290 & 126 & 172 & 206 & 178 & 80 \\
\hline IRRI 154 & 152 & 210 & 270 & 198 & 290 & 128 & 170 & 206 & 190 & 80 \\
\hline FL478 & 152 & 210 & 262 & 198 & 300 & 124 & 170 & 206 & 184 & 80 \\
\hline IR74371 & 154 & $\mathrm{n} / \mathrm{a}$ & 300 & 200 & 300 & 124 & 172 & 204 & 178 & 78 \\
\hline
\end{tabular}

${ }^{\mathrm{a}}$ Marker allele sizes (bp).

${ }^{\mathrm{b}} \mathrm{SUB} 1$ marker alleles from FR13A are shaded in grey.

Table 5. Percentage of polymorphic SNP markers and average PIC value per chromosome.

\begin{tabular}{|c|c|c|c|c|c|c|}
\hline Chr. & Total & $\%$ monomorphic & $\%$ polymorphic & PIC Min & PIC Max & PIC Ave \\
\hline 1 & 45 & 8.9 & 91.1 & 0.045 & 0.531 & 0.314 \\
\hline 2 & 37 & 8.1 & 91.9 & 0.130 & 0.574 & 0.381 \\
\hline 3 & 44 & 13.6 & 86.4 & 0.045 & 0.574 & 0.310 \\
\hline 4 & 31 & 3.2 & 96.8 & 0.087 & 0.588 & 0.342 \\
\hline 5 & 33 & 0.0 & 100.0 & 0.087 & 0.490 & 0.289 \\
\hline 6 & 38 & 2.6 & 97.4 & 0.045 & 0.501 & 0.323 \\
\hline 7 & 28 & 3.6 & 96.4 & 0.087 & 0.527 & 0.319 \\
\hline 8 & 27 & 0.0 & 100.0 & 0.124 & 0.580 & 0.377 \\
\hline 9 & 24 & 0.0 & 100.0 & 0.087 & 0.530 & 0.320 \\
\hline 10 & 19 & 5.3 & 94.7 & 0.087 & 0.517 & 0.382 \\
\hline 11 & 31 & 0.0 & 100.0 & 0.045 & 0.537 & 0.343 \\
\hline 12 & 26 & 3.9 & 96.2 & 0.087 & 0.501 & 0.331 \\
\hline
\end{tabular}


Table 6. Set of genotyping SNPs based on PIC value.

\begin{tabular}{|c|c|c|c|c|c|c|c|}
\hline Chr & SNP & Position (Mb) & PIC Values & Chr & SNP & Position (Mb) & PIC Values \\
\hline 1 & id1003559 & 4.3 & 0.5308 & 7 & id7000461 & 2.7 & 0.4783 \\
\hline 1 & id1004817 & 6.1 & 0.4292 & 7 & id7001478 & 8.2 & 0.4904 \\
\hline 1 & ud1000727 & 16.1 & 0.5267 & 7 & id7002051 & 12.2 & 0.4160 \\
\hline 1 & id1010609 & 19.1 & 0.5174 & 7 & id7002105 & 13.4 & 0.5267 \\
\hline 1 & id1023158 & 38.2 & 0.5290 & 7 & id7002427 & 15.9 & 0.4065 \\
\hline 1 & id1028304 & 44.7 & 0.5015 & 7 & id7002859 & 19.2 & 0.3604 \\
\hline 2 & id2008501 & 22 & 0.5578 & 8 & id8000699 & 2.2 & 0.4368 \\
\hline 2 & id2008866 & 23.2 & 0.4728 & 8 & id8003681 & 12.5 & 0.5552 \\
\hline 2 & id2010969 & 26.1 & 0.4898 & 8 & ud8001072 & 16.6 & 0.5798 \\
\hline 2 & id2011561 & 27.2 & 0.4842 & 8 & id8004838 & 18.4 & 0.5743 \\
\hline 2 & id2014684 & 34 & 0.5743 & 8 & id8006727 & 23.6 & 0.5290 \\
\hline 2 & id 2015767 & 35.8 & 0.5743 & 8 & id 8007472 & 27.4 & 0.4160 \\
\hline 3 & id3000362 & 0.7 & 0.5743 & 9 & id9000661 & 2.3 & 0.3967 \\
\hline 3 & id3005216 & 10.1 & 0.4842 & 9 & id9000881 & 4.2 & 0.4160 \\
\hline 3 & id3008333 & 17.3 & 0.4336 & 9 & id 9003188 & 12.5 & 0.4470 \\
\hline 3 & id3011383 & 27.8 & 0.5229 & 9 & id 9003720 & 14.4 & 0.3845 \\
\hline 3 & id3013669 & 29.9 & 0.4842 & 9 & id9004168 & 15.5 & 0.2970 \\
\hline 3 & id3015399 & 32.9 & 0.3750 & 9 & id9006988 & 20.1 & 0.5298 \\
\hline 4 & ud4000438 & 5.8 & 0.5771 & 10 & id 10000174 & 0.9 & 0.4470 \\
\hline 4 & id4003973 & 13.5 & 0.5629 & 10 & id 10001250 & 4 & 0.5174 \\
\hline 4 & id4007105 & 21.8 & 0.5880 & 10 & id10001624 & 5.2 & 0.4792 \\
\hline 4 & id4007444 & 22.8 & 0.5724 & 10 & wd10001251 & 6.3 & 0.4898 \\
\hline 4 & id4009293 & 28.6 & 0.5880 & 10 & id10005538 & 18.9 & 0.4792 \\
\hline 4 & id4010924 & 32.3 & 0.5015 & 10 & id 10006726 & 21.9 & 0.4292 \\
\hline 5 & id5000015 & 0 & 0.4898 & 11 & id11003924 & 10.8 & 0.5371 \\
\hline 5 & id5001470 & 2.5 & 0.4842 & 11 & id11004398 & 15.1 & 0.4160 \\
\hline 5 & id5001756 & 3 & 0.4336 & 11 & id11004812 & 16.6 & 0.4898 \\
\hline 5 & id5006311 & 15.7 & 0.4551 & 11 & id11005065 & 17.3 & 0.4728 \\
\hline 5 & id5006821 & 17.1 & 0.3604 & 11 & id11005646 & 18.4 & 0.4551 \\
\hline 5 & id5010661 & 23.7 & 0.3658 & 11 & id11008862 & 24.9 & 0.4908 \\
\hline 6 & $\mathrm{fd} 17$ & 6.8 & 0.5015 & 12 & id12000252 & 0.7 & 0.5015 \\
\hline 6 & id6004481 & 7 & 0.3604 & 12 & id12001996 & 4.4 & 0.4677 \\
\hline 6 & id6005350 & 8.2 & 0.4460 & 12 & id12003019 & 7.6 & 0.3026 \\
\hline 6 & id6011018 & 21.8 & 0.4904 & 12 & id12004491 & 12.5 & 0.4393 \\
\hline 6 & id6015793 & 28.5 & 0.4303 & 12 & id12005205 & 14.6 & 0.3967 \\
\hline 6 & id6016490 & 30.1 & 0.4292 & 12 & id 12005501 & 15.7 & 0.4842 \\
\hline
\end{tabular}

(0.490-0.580) which is due to the multi-allelic nature of SSR polymorphism. The average PIC values of SNP markers range from 0.289 to 0.382 (Table 5). SNPs with high PIC values are indicated in Table 6. There were "blind spots" in SNP coverage where the PIC values of the SNP markers were very low especially on chromosomes 5, 7, 9, 10 and 11 (Fig. 1 and Supplementary Fig. 1).

\section{Molecular analysis}

The radial trees generated from SSR and SNP data showed were generally consistent with known pedigrees. Both trees indicated that FR13A and Super Basmati were diverse from the rest of the indica accessions. However, there were differences in the clustering of the 21 indica lines generated from the SSR and SNP analysis. The tree produced from SSR data was more consistent with pedigrees compared to the tree produced from SNP data.

\section{DISCUSSION}

In this study, over 700 SSR markers were selected based on the literature searching for high PIC values and the availability of markers in our labs, and the availability of the 384-plex SNP BeadXpress platform. The plant material used was highly relevant to current breeding activities in 

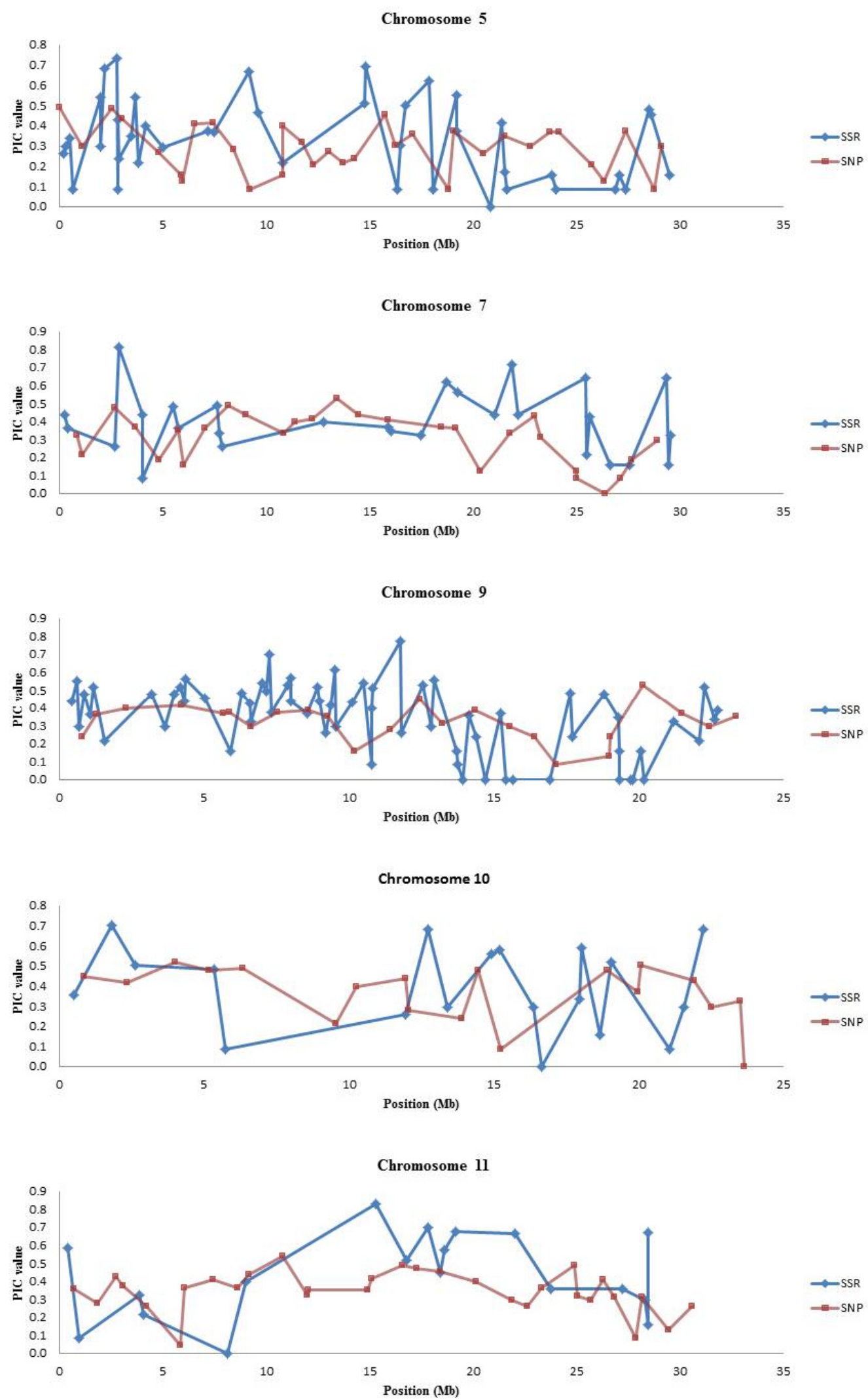

Fig. 1. Graphs of PIC values (y axis) plotted for SSR and SNP markers based on chromosome position (x axis). 
which 21 of the 23 genotypes were indica. This was done to avoid the exaggerated PIC values of markers and to provide a more accurate estimate of the usefulness of a marker in actual breeding programs.

The 12 chromosomes in rice have different sizes (Chen et al. 2002), hence different numbers of markers were screened for different chromosomes. Table 2 shows the uneven distribution of polymorphism of SSR markers between chromosomes. Chromosome 5 was the most polymorphic (80\%) and chromosome 11 is the least polymorphic (53\%). SSRs were identified based on quality of amplification and PIC values, which can be used for MABC, marker-assisted pyramiding and other applications. Specifically for MABC, it is extremely useful to have a set of 'ready to use' highly polymorphic SSRs which we refer to as the indica genotyping set (Table 3). The markers comprising the genotyping set were chosen based on the marker quality, polymorphism and position. Some chromosomes had fewer markers due to lower polymorphism levels in the genotypes that we used. For some molecular breeding applications such as MABC, it is important to have evenly spaced markers with a window of approximately $5 \mathrm{Mb}$ for background selection. Also, 5-10 markers per chromosome are generally sufficient to have an efficient recovery of the recipient parent during background selection or to construct a framework map for QTL analysis.

We emphasized the SUB1 region in this study; hence more markers were screened within and near this locus on chromosome 9. Table 4 shows the markers tightly-linked to $S U B 1$ and compares the allele sizes for comparison between $S U B 1$ and non-SUB1 genotypes. Markers with the highest PIC values will be useful to select markers for tracking $S U B 1$ in indica breeding populations. Polymorphism information is also currently being used to introgress $S U B 1$ into new varieties (Collard et al. 2013; Gregorio et al. 2013).

SNP markers have the potential for providing the highest map resolution (Nasu et al. 2002). SNPs are rapidly replacing SSRs in plant breeding and genetics research because of their abundance and many SNP platforms are high throughput (i.e. many samples per assay) which

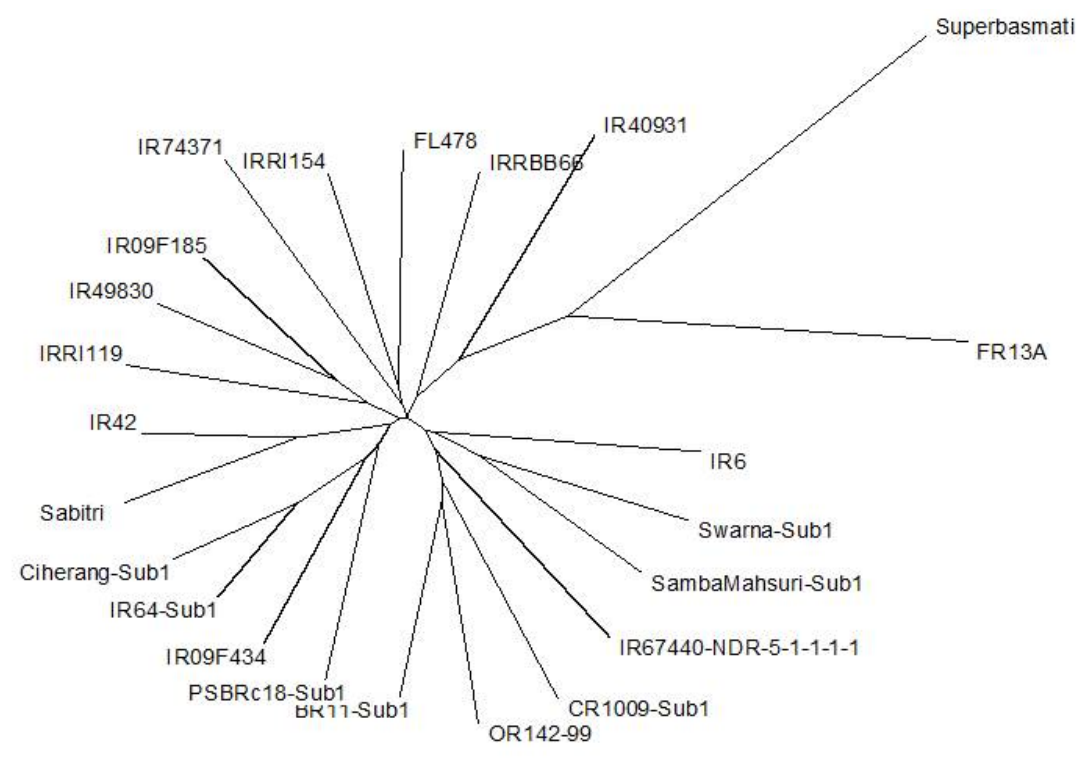

Fig. 2. Radial tree using unrooted neighbor-joining method of SSR data using Nei's similarity coefficient. 
increases efficiency and cost effectiveness (Rafalski 2002;

Duran et al. 2009; Edwards and Batley 2010). An earlier SNP platform assayed 1536 markers but a recent study compared the cost effectiveness of using 96 or 384 SNPs subsets compared to the original one for diversity studies. Results indicated that the 384 SNP markers gave the optimal balance between power and economy for germplasm characterization in barley (Moragues et al. 2010). SNP genotyping (384-plex) using the BeadXpress was used in this experiment due to lower costs per sample (Lin et al. 2009). Table 6 shows the most polymorphic SNP markers based on the PIC values. These SNP markers could also be used in lower-plex SNP genotyping platforms (e.g. 24 or 96 SNPs) such as the Fluidigm EP1 ${ }^{\mathrm{TM}}$ System (Thomson 2014).

SNP platforms such as the BeadXpress platform usually contain a fixed set of SNP markers; hence there may be a need for complementation with other marker systems. Our results indicate that SSR markers can be used to "fill in the gaps" and complement SNP marker genotyping for some molecular breeding applications. Additionally, as shown in Fig. 1 and Supplementary Fig. 1, the 384-plex SNP set has "blind spots" in which markers have very low PIC values; SSRs can specifically be used in these regions for QTL mapping or background selection. For example, on chromosomes 5, 7, 9, 10 and 11, where the SSR markers had higher PIC values compared to SNPs (Fig. 1).

Genetic diversity analysis was also conducted to compare two marker systems. Figures 2 and 3 show radial trees using SSR and SNP markers, respectively. From this analysis, Super Basmati and FR13A were distant to the rest of the lines. However, based on pedigree information, the tree formed using SSR data was more consistent with pedigrees as shown in Table 1. This was probably the result of more SSRs being tested compared to SNPs, and suggests that more markers are needed for genetic diversity analysis to complement with the fixed 384 SNPs when elite indica breeding germplasm is used. Breeding material is more genetically similar compared to diversity panels. The requirement to use more SNP markers compared to SSRs

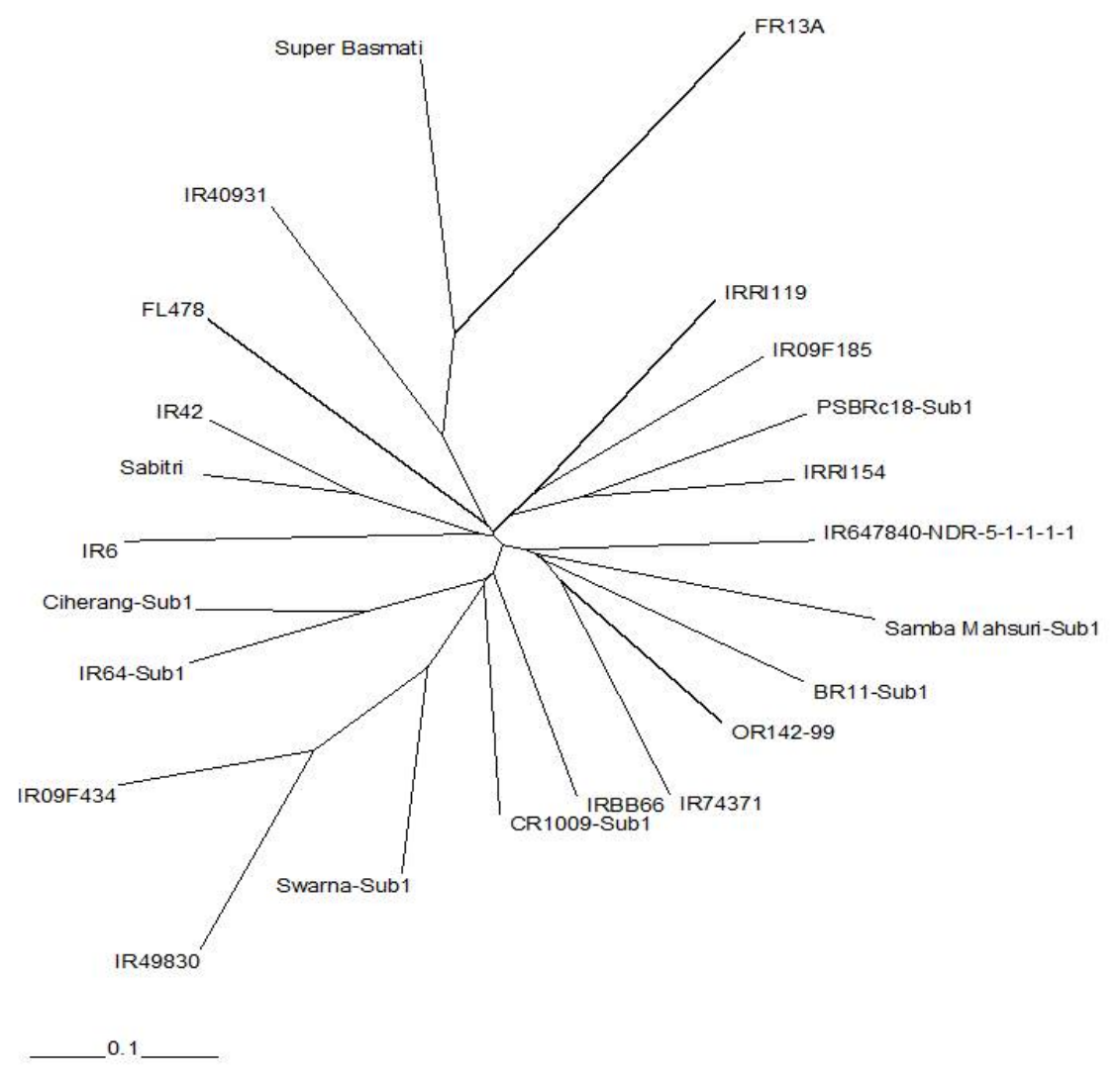

Fig. 3. Radial tree view of unrooted neighbor-joining tree of SNP data using Nei's similarity coefficient. 
for genetic diversity analysis has been previously reported in maize (Hamblin et al. 2007; Yang et al. 2011)

In conclusion, SSRs and SNPs are complementary marker systems that can be used for molecular breeding in rice. SSRs are needed to "fill in the gaps" for fixed SNP arrays like BeadXpress platform, containing the same set of SNP markers for a more efficient and effective process. It is hoped that the genotyping sets identified in this paper will be useful for other rice researchers to efficiently identify polymorphic SSRs or SNPs especially for activities involving indica germplasm.

\section{ACKNOWLEDGMENTS}

The authors gratefully acknowledge funding from the Bill and Melinda Gates Foundation (BMGF) for the Stress Tolerant Rice for Africa and South Asia (STRASA) program. We also thank Dr. Tobias Kretzschmar and Dr. J. Damien Platten (IRRI) for critically reviewing the manuscript.

\section{REFERENCES}

Akagi H, Yokozeki Y, Inagaki A, Fujimura T. 1997. Highly polymorphic microsatellites of rice consist of AT repeats, and a classification of closely related cultivars with these microsatellite loci. Theor Appl Genet 94: 61-67.

Anderson JA, Churchill GA, Autrique JE, Sorrells ME, Tanksley SD. 1993. Optimizing parental selection for genetic linkage maps. Genome 36: 181-186.

Botstein D, White RL, Skolnick M, Davis RW. 1980. Construction of a genetic linkage map in man using restriction fragment length polymorphisms. Am J Hum Genet 32: 314-331.

Chen M, Presting G, Barbazuk WB, Goicoechea JL, Blackmon B, Fang G, Kim H, Frisch D, Yu Y, Sun S, Higingbottom S, Phimphilai J, Phimphilai D, Thurmond S, Gaudette B, Li P, Liu J, Hatfield J, Main D, Farrar K, Henderson C, Barnett L, Costa R, Williams B, Walser S, Atkins M, Hall C, Budiman MA, Tomkins JP, Luo M, Bancroft I, Salse J, Regad F, Mohapatra T, Singh NK, Tyagi AK, Soderlund C, Dean RA, Wing RA. 2002. An integrated physical genetic map of rice genome. Plant Cell 14: 537-545.
Chen X, Temnykh S, Xu Y, Cho YG, McCouch SR. 1997. Development of a microsatellite framework map providing genome-wide coverage in rice (Oryza sativa L.). Theor Appl Genet 95: 553-567.

Cho YG, Ishii T, Temnykh S, Chen X, Lipovich L, McCouch SR, Park WD, Ayres N, Cartinhour S. 2000. Diversity of microsatellites derived from genomic libraries and GenBank sequences in rice (Oryza sativa L.). Theor Appl Genet 100: 713-722.

Collard BCY, Mackill DJ. 2008. Marker-assisted selection : an approach for precision plant breeding in the twentyfirst century. Phil Trans R Soc B 363: 557-572.

Collard BCY, Septiningsih EM, Das SR, Carandang JJ, Pamplona AM, Sanchez DL, Kato Y, Ye G, Reddy JN, Singh US, Iftekharuddaula KM, Venuprasad R, Vera-Cruz CN, Mackill DJ, Ismail AM. 2013. Developing new flood-tolerant varieties at the international rice research institute (IRRI). SABRAO J Breed Genet 45: 42-56.

Duran C, Appleby N, Clark T, Wood D, Imelfort M, Batley J, Edwards D. 2009. AutoSNPdb: an annotated single nucleotide polymorphism database for crop plants. Nucleic Acids Res 37: D951-D953.

Edwards D, Batley J. 2010. Plant genome sequencing: applications for crop improvement. Plant Biotech J 8: 2-9.

Gregorio GB, Islam MR, Vergara GV, Thirumeni S. 2013. Recent advances in rice science to design salinity and other abiotic stress tolerant rice varieties. SABRAO J Breed Genet 45: 31-41.

Hamblin MT, Warburton ML, Buckler ES. 2007. Empirical comparison of simple sequence repeats and single nucleotide polymorphism in assessment of maize diversity and relatedness. PLoS ONE 2(12) e1367.

Iftekharuddaula KM, Newaz MA, Salam MA, Ahmed HU, Mahbub MAA, Septiningsih EM, Collard BCY, Sanchez DL, Pamplona AM, Mackill DJ. 2011. Rapid and highprecision marker assisted backcrossing to introgress the SUB1 QTL into BR11, the rainfed lowland rice mega variety of Bangladesh. Euphytica 178: 83-97.

IRGSP. 2005. The map-based sequence of the rice genome. Nature 436: 793-800.

Jena KK, Mackill DJ. 2008. Molecular Markers and Their Use in Marker-Assisted Selection in Rice. Crop Sci 48: 1266.

Lin CH, Yeakley JM, McDaniel TK, Shen R. 2009. Mediumto high-throughput SNP genotyping using VeraCode microbeads. Methods Mol Biol 496: 129-142. 
Liu K, Muse SV. 2005. PowerMarker: an integrated analysis environment for genetic marker analysis. Bioinformatics 21: 2128-2129.

Lacape JM, Dessauw D, Rajab M, Noyer JL, Hau B. 2007. Microsatellite diversity in tetraploid Gossypium gene pool: assembling a highly informative genotyping set of cotton SSRs. Mol Breed 19: 45-58.

Macaulay M, Ramsay L, Powell W, Waugh R. 2001. A representative, highly informative"genotyping set" of barley SSRs. Theor Appl Genet 102: 801-809.

Mackill DJ, Amante MM, Vergara BS, Sarkarung S. 1993. Improved semidwarf rice lines with tolerance to submergence of seedlings. Crop Sci 33: 749-753.

Mackill DJ, Ismail AM, Singh US, Labios RV, Paris TR. 2012. Development and rapid adoption of submergence-tolerant (Sub1) rice varieties. Adv Agron 115: 303-356.

McCouch SR, Teytelman L, Xu Y, Lobos KB, Clare K, Walton M, Fu B, Maghirang R, Li Z, Xing Y, Zhang Q, Kono I, Yano M, Fjellstrom R, Declerck G, Schneider D, Cartinhour S, Ware D, Stein L. 2002. Development and mapping of 2240 new SSR markers for rice (Oryza sativa L.) (supplement). DNA Res 9: 257-279.

McCouch SR, Zhao K, Wright M, Tung CW, Ebana K, Thomson M, Reynolds A, Wang D, Declerck G, Ali ML, McClung A, Eizenga G, Bustamante C. 2010. Development of genome-wide SNP assays for rice. Breed Sci 60: 524-535.

Moragues M, Comadran J, Waugh R, Milne I, Flavell AJ, Russel JR. 2010. Effects of ascertainment bias and marker number on estimations of barley diversity from highthroughput SNP genotype data. Theor Appl Genet 120: 1525-1534.

Narshimulu G, Jamaloddin M, Vemireddy LR, Anuradha G, Siddiq E. 2011. Potentiality of evenly distributed hypervariable microsatellite markers in marker-assisted breeding of rice. Plant Breed 130: 314-320.

Nasu S, Suzuki J, Ohta R, Hasegawa K, Yui R, Kitazawa N, Monna L, Minobe Y. 2002. Search for and Analysis of Single Nucleotide Polymorphisms (SNPs) in Rice (Oryza sativa, Oryza ruf ipogon) and Establishment of SNP Markers. DNA Res 171: 163-171.

Neeraja CN, Rodriguez RM, Pamplona A, Heuer S, Collard BCY, Septiningsih EM, Vergara G, Sanchez D, Xu K, Ismail AM, Mackill DJ. 2007. A marker-assisted backcross approach for developing submergence-tolerant rice cultivars.
Theor. Appl. Genet. 115: 767-776.

Panaud O, Chen X, McCouch SR. 1996. Development of microsatellite markers and characterization of simple sequence length polymorphism (SSLP) in rice (Oryza sativa L.). Mol Gen Genet 252: 597-607.

Rafalski A. 2002. Applications of single nucleotide polymorphisms in crop genetics. Curr Opin Plant Biol 5: 94-100.

Ribaut J-M, Hoisington D. 1998. Marker-assisted selection: new tools and strategies. Trends Plant Sci 3: 236-239.

Schatz MC, Maron LG, Stein JC, Wences AH, Gurtowski J, Biggers E, Lee H, Kramer M, Antoniou E, Ghiban E, Wright MH, Chia J, Ware D, McCouch SR, McCombie R. 2014. Whole genome de novo assemblies of three divergent strains of rice, Oryza sativa, document novel gene space of aus and indica. Genome Biol 15: 506.

Septiningsih E M, Collard BCY, Heuer S, Bailey-Serres J, Ismail AM, Mackill DJ. 2013. Applying genomics tools for breeding submergence tolerance in rice. In: Varshney RK and Tuberosa R (eds). Translational genomics for crop breeding: Volume 2 - Improvement for abiotic stress, quality and yield improvement. Wiley-Blackwell, USA, 9-30.

Septiningsih EM, Hidayatun N, Sanchez DL, Nugraha Y, Carandang J, Pamplona AM, Collard BYC, Ismail AM, Mackill DJ. 2015. Accelerating the development of new submergence tolerant rice varieties: the case of CiherangSub1 and PSB Rc18-Sub1 Euphytica 202: 259-268.

Septiningsih EM, Pamplona AM, Sanchez DL, Neeraja CN, Vergara GV, Heuer S, Ismail AM, Mackill DJ. 2009. Development of submergence-tolerant rice cultivars: the Sub1 locus and beyond. Ann Botany 103: 151-60.

Shete S, Tiwari H, Elston RC. 2000. On estimating heterozygosity and polymorphism information content value. Theor Popul Biol 57: 265-271.

Singh H, Deshmukh RK, Singh A, Singh AK, Gaikwad K, Sharma TR, Mohapatra T, Singh NK. 2009. Highly variable SSR markers suitable for rice genotyping using agarose gels. Mol Breed 25: 359-364.

Temnykh S, Park WD, Ayres N, Cartinhour S, Hauck N, Lipovich L, Cho YG, Ishii T, McCouch SR. 2000. Mapping and genome organization of microsatellite sequences in rice (Oryza sativa L.). Theor Appl Genet 100: 697-712.

Thomson MJ. 2014. High-Throughput SNP Genotyping to Accelerate Crop Improvement. Plant Breed Biotech 2014: 195-212. 
Thomson MJ, Zhao K, Wright M, McNally KL, Rey J, Tung CW, Reynolds A, Scheflfler B, Eizenga G, McClung A, Kim H, Ismail AM, De Ocampo M, Mojica C, Reveche MY, Dilla-Ermita CJ, Mauleon R, Leung H, Bustamante C, McCouch SR. 2012. High-throughput single nucleotide polymorphism genotyping for breeding applications in rice using the BeadXpress platform. Mol Breed 29: 875-886.

Tung C-W, Zhao K, Wright MH, Ali ML, Jung J, Kimball J, Tyagi W, Thomson MJ, McNally K, Leung H, Kim H, Ahn S-N, Reynolds A, Scheffler B, Eizenga G, McClung A, Bustamante C, McCouch SR. 2010. Development of a Research Platform for Dissecting Phenotype-Genotype Associations in Rice (Oryza spp.). Rice 3: 205-207.

Varshney RK, Baum M, Guo P, Grando S, Ceccarelli S, Graner A. 2010. Features of SNP and SSR diversity in a set of ICARDA barley germplasm collection. Mol Breed 26: 229-242.
Varshney RK, Thiel T, Sretenovic-Rajicic T, Baum M, ValKoun J, Guo P, Grando S, Ceccarelli S, Graner A. 2008. Identification and validation of a core set of informative genic SSR and SNP markers for assaying functional diversity in barley. Mol Breed 22: 1-13.

Wu KS, Tanksley SD. 1993. Abundance, polymorphism and genetic mapping of microsatellites in rice. Mol Gen Genet 241: 225-235.

Xu K, Mackill DJ. 1996. A major locus for submergence tolerance mapped on rice chromosome 9. Mol Breed 2: 219-224.

Yang X, Xu Y, Shah T, Li H, Han Z, Li J, Yan J. 2011. Comparison of SSRs and SNPs in assessment of genetic relatedness in maize. Genetica 139: 1045-1054.

Zheng KL, Subudhi PK, Domingo J, Magpantay G, Huang N. 1995. Rapid DNA isolation for marker assisted selection in rice breeding. Rice Genet News 12: 255-258. 